San Jose State University

SJSU ScholarWorks

Faculty Publications

Electrical Engineering

2009

\title{
A Trade-off Analysis of Energy Detectors and Partitioned Search for Primary Detection
}

Birsen Sirkeci-Mergen

San Jose State University, birsen.sirkeci@sjsu.edu

Vishal Sawant

San Jose State University

Robert H. Morelos-Zaragoza

San Jose State University, robert.morelos-zaragoza@sjsu.edu

Follow this and additional works at: https://scholarworks.sjsu.edu/ee_pub

Part of the Electrical and Computer Engineering Commons

\section{Recommended Citation}

Birsen Sirkeci-Mergen, Vishal Sawant, and Robert H. Morelos-Zaragoza. "A Trade-off Analysis of Energy Detectors and Partitioned Search for Primary Detection" Faculty Publications (2009): 1-4. https://doi.org/ 10.1109/ICCCN.2009.5235213

This Article is brought to you for free and open access by the Electrical Engineering at SJSU ScholarWorks. It has been accepted for inclusion in Faculty Publications by an authorized administrator of SJSU ScholarWorks. For more information, please contact scholarworks@sjsu.edu. 


\title{
A Trade-off Analysis of Energy Detectors and Partitioned Search for Primary Detection
}

\author{
Birsen Sirkeci-Mergen, Vishal Sawant, Robert H. Morelos-Zaragoza \\ Electrical Engineering Department \\ San Jose State University \\ San Jose, California 95192-0084 \\ Email: \{bsirkeci@email.sjsu.edu, vishu.s21@gmail.com, Robert.Morelos-Zaragoza@sjsu.edu\}
}

\begin{abstract}
Cognitive radios aim to coexist in the unused spectrum bands which are licensed to primary users without harming the primary transmission/reception. For a cognitive radio, it is important to detect the band in which the primary is operating as fast as possible and with high reliability in order to adapt its transmission. In this work, we propose P-partitioning method in combination with energy detectors for the search of the band that the primary user is operating. In the P-partitioning method, the spectrum bands are categorized into $P$ groups and the group that the primary band belongs to is detected in a recursive fashion. The energy detector operates on each group and the test statistics is the total energy received in the bands belonging to the group. The proposed search technique has detection time $P \log _{P}(N)$, where $N$ is the number of bands in the spectrum. When $P=N$, the proposed scheme is equivalent to linear search with detection time $N$.

We study the performance of the proposed scheme for a single non-cooperative radio and also for multiple cooperating radios. For a single cognitive radio, we provide an upper bound on the probability of correct detection which presents two different regimes of operation. In the low SNR regime, although it is counter-intuitive the partitioning improves the probability of detection. This is due an averaging effect when the signal energy in different bands are accumulated to obtain the energy contribution from a group. In the high SNR regime, performance degrades with partitioning. In addition, we observe that user cooperation improves the performance in the high SNR regime.
\end{abstract}

\section{INTRODUCTION}

Recently, there has been tremendous interest in designing cognitive radios which are capable of detecting and operating in the under-utilized frequency bands [1]-[3]. Detection of these bands at different places and at different times is a fundamental problem and requires additional insight over the traditional way of signal detection in a point-to-point link. Cognitive radios, also known as secondary users, should operate without disturbing the transmission/reception of the primary user; hence they should be capable of reliable and fast detection of the band that primary is using.

The already existing primary detection methods can be grouped into three: (i) energy detection; (ii) pilot detection; and (iii) feature detection. Energy detectors are non-coherent and relatively simple; however may not be as accurate as the feature based detectors. There has been various implementation of energy detectors both analog and digital. The analog energy detectors require either filter banks or tunable filters to adjust to the bandwidth of interest. Digital energy detectors require wide-band ADC. There are also more advanced methods such as MRSS which is a digitally-assisted analog energy detection method [4]. In any of the above implementations, it is critical to reduce the time spent by the energy detectors for a prompt estimate of the occupied bands.

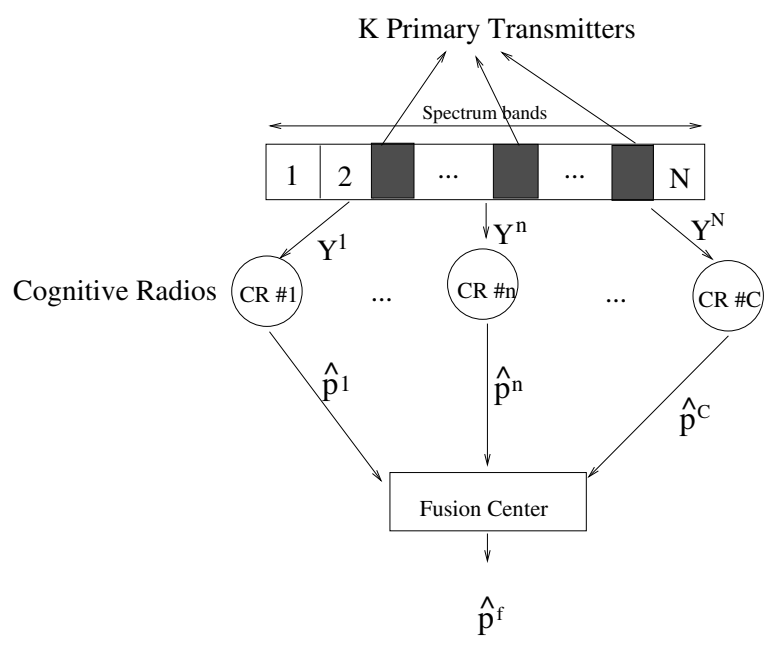

Fig. 1. System Model: $\mathbf{Y}_{i}=\left[Y_{1}^{(i)} Y_{2}^{(i)} \ldots Y_{N}^{(i)}\right]$ denotes the received vector at the $i^{\prime}$ th cognitive radio, $\hat{p}^{(i)}$ is the estimate of the band primary operating at the $i$ 'th node.

In this work, we study the P-partitioning method for the search of the band that the primary is operating. In the considered set-up, each cognitive radio divide the spectrum into $\mathrm{P}$ groups and the group which has highest total energy is selected as the candidate group. In the next iteration, the candidate group is divided into $\mathrm{P}$ groups and the search for the primary band continues recursively until the candidate group has a single element which is the estimate for the primary band. When $P=N$, this is equivalent to linear searching for the band with the highest energy among $N$ bands. The detection time of the P-partitioning method is proportional $P \log _{P}(N)$ and is minimum when $P=2$ for a given $N$.

We are interested in the effect of partitioning to the probability of correct detection. Interestingly, we observe two different regimes of operation. In the low SNR regime, the best performance is observed when $P=2$, whereas in the 
high SNR regime, the performance gets better as $P$ increases. In other words, partitioning helps improve the performance in the low SNR regime. In the high SNR regime, better detection time can be traded off for the loss in the performance.

Different search techniques for detection of primary and empty spectrum bands has been analyzed in [5]-[8]. In [5], the authors study various algorithms to solve the channel searching problem by modelling it as a Markovian decision process. In [6], the authors analyze the performance of random and serial search techniques in terms of mean detection time. They show that n-step serial search has lower detection time than random search. In [7], authors propose a two-stage (coarse and fine) sensing technique for dynamic spectrum access. The n-step serial search and two-stage sensing technique [6], [7] are close to P-partitioning search in the sense that they all have decreased detection time due to grouping. Different from previous work, our goal is to try to identify the tradeoffs between detection time and detection probability. In [8], the authors provide a dynamic programming solution for determining the optimal sensing order in sequential search. In addition, cooperative sensing has also been studies in [9][11].

The rest of this paper is organized as follows. Detailed descriptions of system model and proposed schemes are provided in Section II. We present the analysis of probability of detection for a single cognitive radio in Section III and in Section IV we describe the considered user cooperation among the cognitive radios. Simulation results are provided in Section $\mathrm{V}$ for both single non-cooperative and cooperative radios. We conclude the paper in Section VI.

\section{System Model}

We consider a network formed by multiple cognitive radios and a single primary transmitter (see Fig. 1). The spectrum is assumed to be divided into $N$ bands and the primary is operating in one of the $N$ bands. Without loss of generality let's assume the primary is operating in band $p=1$. Let $C$ denote the number of cognitive radios in the network. The received signal at the $i$ 'th cognitive radio in the $k$ 'th band is

$$
R_{k}^{(i)}= \begin{cases}N_{k}^{(i)} & k \neq p \\ N_{p}^{(i)} & k=p\end{cases}
$$

where $\left\{N_{k}^{(i)}\right\}$ s for $i=1 \ldots C, k=1 \ldots N$ are assumed to be Gaussian and independent between bands and cognitive radios. We assume $N_{k}^{(i)} \sim \mathcal{N}_{c}\left(0, \sigma^{2}\right)$ and $N_{p}^{(i)} \sim \mathcal{N}_{c}\left(0, \sigma_{p}^{2}\right)$. The signal-to-noise ratio (SNR) is defined as

$$
S N R=\sigma_{p}^{2} / \sigma^{2}
$$

For primary detection, each cognitive radio uses the average statistics over an interval of $M$ samples:

$$
Y_{k}^{(i)}=\sum_{n=1}^{M}\left|R_{k}^{(i)}[n]\right|^{2}, \quad k=1 \ldots N, i=1 \ldots C .
$$

The $i$ 'th cognitive radio has access to $Y_{k}^{(i)}, k=1 \ldots N$, and utilize the energy detector and $P$ partitioning search in order to decide which band has the primary signal. Without loss of generality, we assume $N$ is a power of $P$, i.e. $N=P^{K}$. For the P-partitioning search, the estimate of the primary band, $\hat{p}^{(i)}$, is determined via the following recursive method:

0) Let $S=\{1, \ldots, N\}$.

1) Divide the $S$ into $P$ consecutive partitions $S_{1}, S_{2}, \ldots, S_{P}$

2) Find the partition that has maximum total energy:

$$
\hat{p}=\arg \max _{k \in\{1, \ldots, P\}} \sum_{m \in S_{k}} Y_{m}^{(i)}
$$

3) Let $S=S_{\hat{p}}$ and continue from step 1 if $|S|>1$; otherwise $S=\left\{\hat{p}^{(i)}\right\}$ contains the estimate for the primary band.

When $P=N$, the decision of the band number for primary at the $i$ 'th node is given as

$$
\hat{p}^{(i)}=\arg \max _{k \in S} Y_{k}^{(i)}
$$

where $S=\{1, \ldots, N\}$ is the set of band indexes.

\section{PERFormance of P-PARTITIONING SEARCH}

The time spent by each cognitive radio to complete the search via the proposed P-partitioning method is

$$
T^{(i)} \propto P \times \log _{P}(N) .
$$

Note that $2 \leq P \leq N$, hence the best time is achieved when $P=2$.

The probability of correct detection for each cognitive radio is in the form

$$
\begin{aligned}
P_{d}^{(i)} & :=\operatorname{Pr}\left\{\hat{p}^{(i)}==p\right\} \\
& =\operatorname{Pr}\left(\bigcap_{n=1}^{\log _{P}(N)} \bigcap_{j=1}^{P-1}\left\{\sum_{k=1}^{P^{n-1}} Y_{k}^{(i)} \geq \sum_{k=j P^{n-1}+1}^{(j+1) P^{n-1}} Y_{k}^{(i)}\right\}\right)
\end{aligned}
$$

The explicit derivation of Eqn. 2 is not tractable due to the dependence of the event $\left\{\sum_{k=1}^{P^{n-1}} Y_{k}^{(i)} \geq \sum_{k=j P^{n-1}+1}^{(j+1) P^{n-1}} Y_{k}^{(i)}\right\}$ for different $j$ and $n$ values. Instead, we derive an upper bound on the probability of correct detection $P_{d}^{(i)}$. In Section $\mathrm{V}$, we show that the provided upper bound have similar characteristics to the actual $P_{d}^{(i)}$ vs $S N R$ curves. Our goal is to understand the effect of partitioning parameter $P$ and $S N R$ on the probability of correct detection.

It is well-known that probability of intersection of events is less than the minimum of the individual probabilities, i.e.

$$
P\left(A_{1} \cap A_{2}\right) \leq \min _{k=1,2} P\left(A_{k}\right) .
$$

Using this property, we obtain

$$
P_{d}^{(i)} \leq \min _{j, n} \operatorname{Pr}\left\{\sum_{k=1}^{P^{n-1}} Y_{k}^{(i)} \geq \sum_{k=j P^{n-1}+1}^{(j+1) P^{n-1}} Y_{k}^{(i)}\right\} .
$$


Note that $Y_{k}^{(i)}=\sum_{n=1}^{M}\left|R_{k}^{(i)}[n]\right|^{2}$ is a chi-square distributed random variable:

$$
\begin{aligned}
& Y_{k}^{(i)} \sim \sigma^{2} \mathcal{X}_{2 M}^{2} \quad \text { if } k \neq p \\
& Y_{k}^{(i)} \sim \sigma_{p}^{2} \mathcal{X}_{2 M}^{2} \quad \text { if } k=p
\end{aligned}
$$

From the central limit theorem, for large $M$ values, we can approximate the chi-square distribution $\mathcal{X}_{M}^{2}$ with a Gaussian distribution with mean $M$ and variance $2 M$. Using this approximation for large $M$ values, we get

$$
\begin{array}{ll}
Y_{k}^{(i)} \sim \mathcal{N}\left(\sigma^{2} 2 M, \sigma^{4} 4 M\right) & \text { if } k \neq p \\
Y_{k}^{(i)} \sim \mathcal{N}\left(\sigma_{p}^{2} 2 M, \sigma_{p}^{4} 4 M\right) & \text { if } k=p
\end{array}
$$

Let $A_{j n}:=\sum_{k=1}^{P^{n-1}} Y_{k}^{(i)}-\sum_{k=j P^{n-1}+1}^{(j+1) P^{n-1}} Y_{k}^{(i)}$. Under the Gaussian approximation of chi-square distribution, $A_{j n}^{(i)} \sim$ $\mathcal{N}\left(\mu, \sigma_{n}^{2}\right)$ where

$$
\begin{gathered}
\mu=2 M\left(\sigma_{p}^{2}-\sigma^{2}\right) \\
\sigma_{n}^{2}=\sigma_{p}^{4} 4 M+\sigma^{4} 4 M\left(2 P^{n-1}-1\right) .
\end{gathered}
$$

Note that the distribution of $A_{j n}$ does not depend on $j$. Hence, for large $M$,

$$
P_{d}^{(i)} \leq \min _{n} \operatorname{Pr}\left\{A_{j n} \leq 0\right\}=\min _{n} Q\left(\frac{-\mu}{\sigma_{n}}\right),
$$

where $Q(x)=\int_{x}^{\infty} \frac{1}{\sqrt{2 \pi}} e^{-t^{2} / 2} d t$. Using the definition $S N R=$ $\sigma_{p}^{2} / \sigma^{2}$, we obtain

$$
\begin{aligned}
P_{d}^{(i)} & \leq \min _{n} Q\left(\frac{-\mu}{\sigma_{n}}\right) \\
& =\min _{n} Q\left(\frac{\sqrt{M}(1-S N R)}{\sqrt{S N R^{2}+2 P^{(n-1)}-1}}\right)
\end{aligned}
$$

Since $Q(x)$ is a decreasing function, the $P_{d}^{(i)}$ is upper bounded as

$$
P_{d}^{(i)} \leq Q\left(\frac{\sqrt{M}(1-S N R)}{\sqrt{S N R^{2}+2 N P^{-1}-1}}\right) .
$$

Note that the above upper bound is obtained when $n=$ $\log _{P}(N)$.

It is important to note that the $P_{d}$ has two different characteristic based on the region of operation: (i) low SNR regime; (ii) high SNR regime. In the low SNR regime, that is if $S N R<1$, it is better to partition the bands and best performance obtained when $P=2$. This may sound counterintuitive at first since the noise is accumulated at each partition; however in the low SNR regime it becomes critical to average out the effect of noise which can be achieved by partitioning. On the other hand, if $S N R>1$, then partitioning does harm the performance due to accumulation of noise in each partition. In this regime, optimal $P$ is equal to $N$ which implies no partitioning. However, it is important that partitioning reduces the sensing time (see Eqn. 1). In the next section, we consider cooperating cognitive radios in order to improve the performance.

\section{CoOperation AMONG COGNitive RAdios}

We envision a distributed cooperation scheme among cognitive radios in order to eliminate the need of any extra overhead for coordination. In the simple cooperation scheme we consider, each cognitive radio sends their estimate for the primary band number. We assume in the link from the cognitive radio to the fusion center, reliable communication is possible since few bits are transmitted. In other words, we assume there is no noise in the link from the cognitive radios to the fusion center.

The fusion center chooses the most repeated decision among all the cognitive radios:

$$
\hat{p}^{f}=\operatorname{mode}\left\{\hat{p}^{(1)}, \hat{p}^{(2)}, \ldots, \hat{p}^{(N)}\right\}
$$

The radios can transmit using orthogonal channel access techniques such as TDMA, FDMA or CDMA. In this case, we need $C$ orthogonal channels. In addition, the radios can transmit on $B$ orthogonal channels, and use the channel corresponding to their estimate. In this way, the fusion centers receives an accumulated energy in the band that is mostly chosen by the cognitive radios and can simply choose the highest energy band as its estimate. Depending on the value of $B$ and $C$, the above described schemes can be preferred to the other.

On the other hand, cognitive radios can transmit further information such as the energy in the estimated band. This will waste the resources; however it might improve the performance at the fusion center. In this paper, we are interested in the effect of partitioning at the cognitive radios in combination with a simple user cooperation.

\section{Simulations}

In this section, we present the performance of the proposed P-partition method in combination with energy detectors. We obtain the average probability of detection through MonteCarlo methods and validate the conclusions we draw in the analytical sections.

In Fig. 2, we plot the probability of detection for a single cognitive radio as a function of the $S N R=\sigma_{p}^{2} / \sigma^{2}$. The simulation parameters $N=64, M=2$, and $P=2,8,64$. The curves has similar characteristic to the upper bound we obtain in Section III. There exists a breakpoint at $S N R=0 d B$ below which using partitioning is advantage and above which partitioning harms the performance. It is also important to note that, the analytical characterization was obtained for large $M$ values and simulation results have similar characteristics even for $M=2$.

In Fig. 3, we plot the probability of detection at a fusion center as a function of the $S N R=\sigma_{p}^{2} / \sigma^{2}$. The simulation parameters $N=64, M=2, P=2,64$, and the number of cognitive radios $C=4,8$. It is important to note that cooperation among cognitive radios improves the performance in the high SNR regime only. However, we believe this result strongly depends on the channel modeling between the cognitive radios and the fusion center. In the case of fading and noise, the user cooperation becomes more critical. 


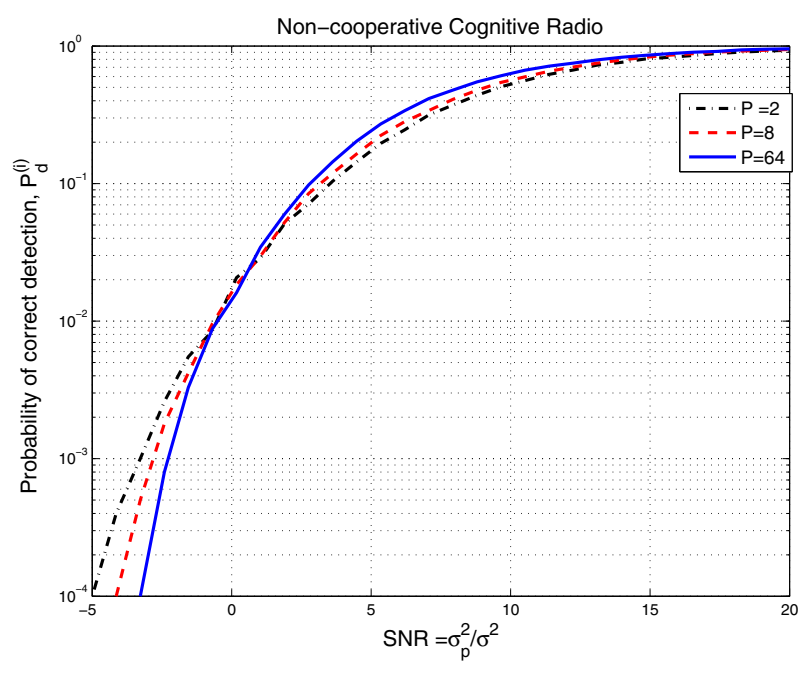

Fig. 2. Single Non-cooperative Cognitive Radio:

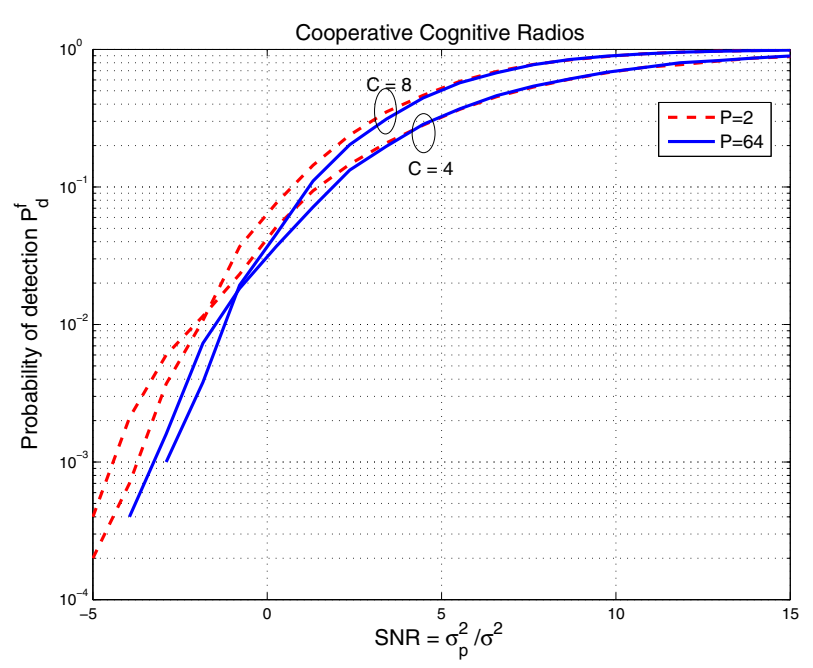

Fig. 3. Multiple Cognitive Radios

\section{CONCLUSION}

In this paper, we analyzed the energy detector in combination with a P-partitioning search for detection of the primary band. Both analytically and by simulations, we showed that partitioning effects the performance depending on which SNR regime (low or high) the radios operate. Furthermore, partitioning decreases the detection time when compared with classical linear search.

The proposed P-partitioning search is simple and effective in detecting a single primary transmitter. We extend the proposed scheme to detection of empty bands in the case of multiple primary transmitters in [12].

\section{REFERENCES}

[1] S. Haykin, "Cognitive radio: Brain-empowered wireless communications," IEEE J. Select. Areas Commun., Vol.23, Issue 2, pp.201-220, Feb. 2005.
[2] J. Mitola and G. Maguire, “Cognitive radio': Making software radios more personal," IEEE Personal Communications, Vol.6, Issue 4, pp. 13 18,Aug. 1999.

[3] D. B. Cabric, "Cognitive radios: System design perspective," Ph.D. dissertation, UC Berkeley, CA, Fall 2007.

[4] J. Jongmin Park; Kwan-woo Kim; Taejoong Song; Sang Min Lee; Joonhoi Hur; Kyutae Lim; Laskar, "A cross-layer cognitive radio testbed for the evaluation of spectrum sensing receiver and interference analysis," in 3rd International Conference on Cognitive Radio Oriented Wireless Networks and Communications, 2008. CrownCom 2008., 15-17 May 2008 Page(s): 1 - 6.

[5] Y. Sixing and L. Shufang, "Optimization for time consumption on channel searching in cognitive radio system with markov decision processes," in 4th International Conference on Wireless Communications, Networking and Mobile Computing, 2008. WiCOM '08., 2008.

[6] L. Luo and S. Roy, "Analysis of search schemes in cognitive radio," in 4th Annual IEEE Communications Society Conference on Sensor, Mesh and Ad Hoc Communications and Networks, 2007. SECON '07., 2007.

[7] - "A two-stage sensing technique for dynamic spectrum access," in ICC 2008., 2008.

[8] R. F. Hai Jiang, Lifeng Lai and H. V. Poor, "Cognitive radio: how to maximally utilize spectrum opportunities in sequential sensing," in IEEE Global Communications Conferences(Globecom), Nov. 30- Dec. 4, 2008., 2008.

[9] J. Unnikrishnan and V. Veeravalli, "Cooperative sensing for primary detection in cognitive radio," IEEE Journal of Selected Topics in Signal Processing, Feb 2008.

[10] O. S. A. Betran-Martinez and Y. Bar-Ness, "Detecting primary transmitters via cooperation and memory in cognitive radio," in Information Sciences and Systems, 2007. CISS '07, 2007.

[11] U. S. J. Gambini, O. Simeone and Y. Bar-Ness, "Cooperative cognitive radios with optimal primary detection and packet acceptance control," in IEEE 8th Workshop on Signal Processing Advances in Wireless Communications, 2007. SPAWC 2007., 2007.

[12] B. Sirkeci-Mergen, V. Sawant, and R. H. Morelos-Zaragoza, "A trade-off analysis of energy detectors and partitioned search for empty spectrum detection," submitted to IEICE Transactions on Communications, Special Section on Dynamic Spectrum Access, 2009. 